\title{
Revisión de los procesos de comprensión y producción de textos académicos en el ámbito de la educación superior presencial y virtual
}

\author{
Sandra Acevedo ${ }^{1}$ \\ Lizedh Martínez ${ }^{2}$ \\ Ivonne Román ${ }^{3}$
}

\begin{abstract}
Resumen
Este artículo es producto de una indagación sobre investigaciones y experiencias acerca de los procesos de comprensión y producción de textos en educación superior a nivel internacional y nacional. En los artículos encontrados se destacan, trabajos de investigación, experiencias, pruebas piloto obtenidas a través de cursos de corta duración sobre lectura y/o escritura.
\end{abstract}

Se lograron identificar algunas dificultades que presentan los estudiantes al ingreso en los primeros semestres de vida universitaria. Primero, los imaginarios de los maestros respecto a la escolaridad previa de sus estudiantes; segundo, se identificaron estrategias pedagógicas que se han implementado, y cómo esto aporta elementos fundamentales para representar un buen desempeño en el futuro campo profesional.

Palabras clave: Comprensión de textos y producción de textos, lectura y escritura en educación superior. 


\title{
Review the processes of understanding and production of academic texts in the field of higher education in the classroom and virtual enviroment
}

\begin{abstract}
This article is a product of an investigation about some researchings about the processes of reading comprehension and production of texts in high education to a national and international level. The founded articles detach some searching experiences that start as a pilot trials and practices obtained by mean of short courses about reading comprehension and writing.

It was possible to identify some difficulties that students have when they arrive to the first levels of their university lives; teachers have some imaginary ideas about the experiences their students had in their previous studies, and the pedagogical strategies that they have implemented, and how this add some basis elements to represent a performance in their future professional activity.
\end{abstract}

Keywords: Reading comprehension and texts production, reading and writing in high level education.

Recibido: 09/10/2014

Aceptado: 03/12/2014

\section{Introducción}

Este artículo, documenta el resultado de un proceso de indagación, sistematización y análisis que se dio a partir de artículos publicados en revistas electrónicas sobre experiencias e investigaciones realizadas, en relación a los procesos de comprensión y producción de textos académicos en educación superior. Al rastrear la bibliografía publicada y situarla con fines analíticos en este articulo, se identificaron algunas subcategorías y relaciones establecidas para lograr una mejor descripción y sistematización. Vale la pena aclarar que las investigaciones publicadas fueron tomadas en español y recogen principalmente experiencias colombianas y algunas de países de Iberoamérica. 
Por tanto, la descripción se presenta según dos grandes categorías: lectura y escritura. Y para facilitar la comprensión de la estructura categorial y relacional se presenta la siguiente gráfica:

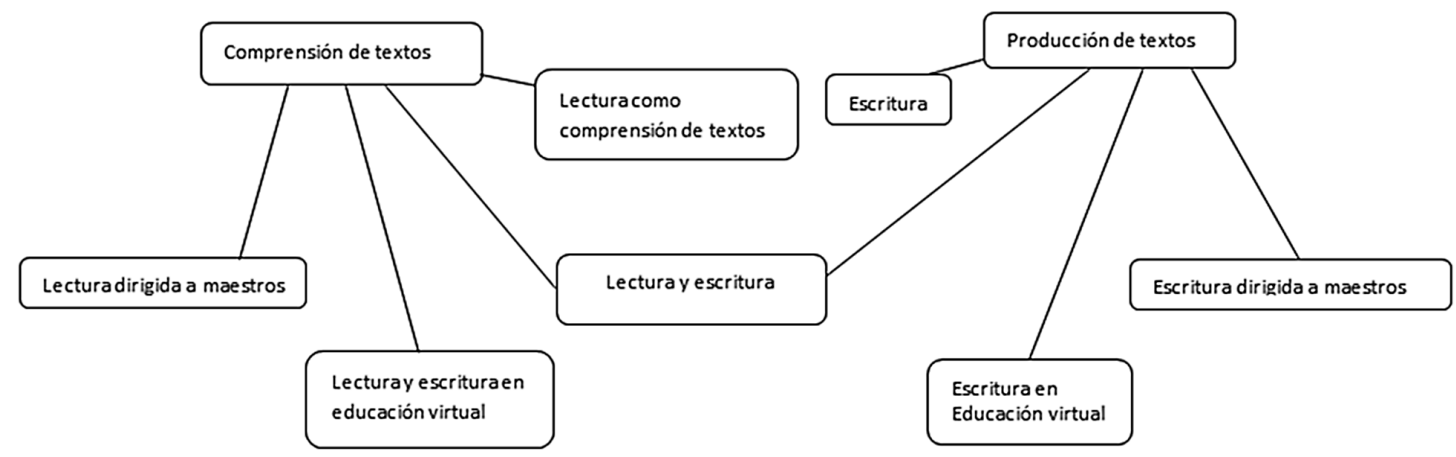

Figura 1. Estructura categorial y relacional.

Fuente: Propia.

\section{Metodología}

Se desarrolló un proceso de revisión sobre una muestra de 50 artículos de investigación y experiencias relacionadas con los procesos lecturas y escritura, y/o la comprensión y producción de textos en educación superior. Para definir dicha muestra, se realizó inicialmente un proceso de rastreo documental; luego, una selección de artículos en diferentes bases de datos, revistas y blogs nacionales e internacionales. La etapa siguiente, fue la sistematización en un proceso de lectura, tematización y registro en matriz categorial; esto permitió analizar las categorías y algunas relaciones que se describen como fruto de los elementos aportados por estas investigaciones y experiencias, acerca de cómo han sido abordados los procesos de comprensión y producción de textos académicos con estudiantes de educación superior.

\section{Desarrollo}

El análisis realizado de las investigaciones sobre comprensión y producción de textos académicos, permitió identificar la presencia en educación superior, de un mayor número de experiencias en modalidad presencial, en comparación a la producción en modalidad virtual y a distancia. Por tanto, siguiendo la estructura propuesta en este artículo, se presentan los siguientes apartes como agrupación fruto del análisis realizado: comprensión de textos y estrategias cognitivas, comprensión de textos virtuales, el maestro como mediador en la experiencia 
de comprensión, lectura y escritura en la universidad, lectura y escritura en educación virtual y a distancia, escritura y lectura dirigido a maestros.

\section{Comprensión de textos y estrategias cognitivas}

¿Cuáles son los interrogantes que movilizan una investigación en relación a los procesos de lectura? Para responder a esta pregunta, Rinaudo (1991), da cuenta de algunos problemas: ¿Cuál es la índole de las dificultades que tienen los estudiantes universitarios, especialmente durante el primer año, para aprender significativamente a partir de texto escrito?; ¿Cómo se deberían orientar los aprendizajes para mejorar sus niveles de rendimiento académico?; ¿La enseñanza secundaria, contribuye al desarrollo de las estrategias cognoscitivas necesarias para las tareas de aprendizaje de textos que se requieren en la universidad?

Ahora bien, para retomar un marco desde el cual sea posible abordar una intervención en la comprensión lectora; Sánchez, Orrantia y Rosales (1992), consideran oportuno; primero, determinar lo que implica «el comprender», antes de hablar sobre comprender un texto. Segundo, reflexionar sobre el cómo evaluar e instruir en este campo. Tercero, se describe un programa de instrucción orientado a la mejora de las habilidades implicadas en la comprensión de textos. De esta forma, el ejercicio permitió demostrar que el ser humano, duplica el significado del texto en la mente y construye un mundo a partir del texto.

Sin embargo, ¿Que es lo que impide al alumno tener una buena comprensión lectora? Oliva (1999), plantea que el alumno no tiene buena comprensión de lectura, ya qué no desarrolla un determinado método. Ésta investigación muestra, cómo se puede enseñar a ser lector crítico a través de metodologías de análisis científico, brindándole al estudiante herramientas necesarias para una excelente comprensión de textos y mejoramiento de comunicación, por medio de clases individuales y en pequeños grupos.

A propósito de estrategias cognitivas, Maturano, Soliveres, y Macías (2002), dan a conocer los resultados de una investigación realizada sobre algunas estrategias cognitivas y meta-cognitivas que usan los alumnos universitarios de diferentes carreras. En general, la comprensión del texto es limitada y, por lo tanto, no está acorde con lo esperado para alumnos de este nivel, en cuanto a su manera de comprender y extraer información de un texto expositivo; el porcentaje de alumnos que manifiesta haber encontrado contradicción en el texto, es inferior a los que indican haber tenido obstáculos en su comprensión.

Sumado a los estudios que pretenden responder ¿Porqué los alumnos de educación superior no tienen buena comprensión de lectura? Rivera (2003), observa conversaciones con alumnos y profesores, acerca de las características de la comprensión lectora en alumnos de tercer y cuarto año de enseñanza media y de primer año universitario. De esta forma, logró identificar mayor fluidez, coherencia y ampliación del vocabulario, cuando los alumnos se concentran en 
explicar las asociaciones de los diagramas realizados en grupo y esto influye en la calidad de la exposición porque aumenta la seguridad en la organización de las ideas.

Si bien es cierto, que la competencia lectora de los estudiantes que ingresan a las universidades colombianas no son las más adecuadas, Silvera, Collante, Tarazona y Ortiz (2003), proponen a través de un programa coherente con la situación, producir efectos favorables, soportándose en la teoría del «Lector Modelo» de Umberto Eco. Para los autores, el problema de la lectura no se sitúa en el terreno de las personas sino de los textos que estas producen. «No se trata, por ejemplo, de entender a García Márquez, sino las obras de García Márquez». Es decir, que el lector no solo comprenda sino también, que sea capaz de criticar la lectura a partir de los propósitos de la misma y los que se hallan de manera explícita o implícita en ellos, no en lo que el citado autor ha pensado, dicho o hecho cotidiana e históricamente.

De otra parte, para profundizar en el funcionamiento meta-cognitivo; respecto al nivel de comprensión de estudiantes universitarios que realizan lecturas de artículos científicos, Ochoa y Aragón (2005), a través de un ejercicio de lectura y reseña de dos tipos de artículos científicos, encontraron una correlación significativa y positiva entre el funcionamiento meta-cognitivo y los niveles de comprensión: a mayor nivel de funcionamiento meta-cognitivo, mayor nivel de comprensión lectora y a menor nivel de funcionamiento meta-cognitivo, menor nivel de comprensión. Igualmente observaron en las grabaciones, que durante el proceso de planificación los estudiantes no regulados empezaron a leer directamente tanto el ensayo teórico como el reporte de investigación, sin detenerse antes a observar los títulos y la longitud del mismo.

De esta forma, se hace evidente ante la realidad tecnologizada, el desarrollo y evolución del conocimiento; como bien lo señalan Sabaj y Ferrari (2005), una demanda sobre la necesidad de comprender y manejar textos escritos (en papel) como aspecto esencial para el desenvolvimiento, tanto en los ámbitos académicos como profesionales. Además, develan que ciertos niveles y procesos de comprensión resultan sencillos según el tipo de sujeto, teniendo en cuenta que «lo literal es más simple que lo inferencial y lo local más fácil que lo global».

De otra parte, también se identifica que las dificultades en la lectura no residen en la enseñanza o en el esfuerzo de los estudiantes por aprender, sino que son debidas a que un porcentaje apreciable, no ha alcanzado el nivel intelectual de las operaciones formales; tales como, el nivel de pensamiento. Para Lucero, Vitale, Iturralde, Valente y Mazzitelli (2006), de acuerdo con los estudios del desarrollo cognitivo en la juventud, el joven se caracteriza por la capacidad de pensar y razonar fuera de su mundo real y de sus propias creencias, puesto que, en esta etapa, la cognición se apoya en un simbolismo puro en el uso de proposiciones, antes, que en la realidad. 
Así pues, Echevarría (2006), propone que la relación «dificultades de la lectura como formación de un hábito», podría estimular la lectura frecuente en los alumnos universitarios. Además, considera que la comprensión de textos implica un conglomerado de procesos, cuya activación o funcionamiento, permite una comprensión de lectura más o menos profunda, superficial, o no comprensiva;, entre los que se destacan: Micro, macro, superestructura y modelo de situación, con el fin de extraer las ideas expresadas en palabras y oraciones del texto, para realizar inferencias que garanticen relaciones de coherencia.

Asimismo, surge el interés en develar si el procesamiento realizado por el lector durante la comprensión de un discurso determinado se mantiene o cambia según la naturaleza del discurso. Por su parte, Escudero y León (2007), proponen: primero, la cognición causal como una característica inherente y por tanto universal del pensamiento humano; segundo, la comprensión como una actividad inteligente y muy relacionada con dicha cognición causal, que requiere de un nivel de coherencia local y global con un fuerte contenido causal; y tercero, que para desarrollar tal actividad se requiere necesariamente de inferencias causales, encargadas de tender puentes de conocimiento entre la información entrante y la que posee el lector, a fin de establecer la coherencia necesaria en la comprensión final.

Así pues, una puesta en práctica realizada por Miljanovich, Huerta, Atalaya, Evangelista, Chávez, Castañeda, Paredes y Woll (2007), demuestra la posibilidad de construir un modulo de lectura a partir de un fundamento teórico de enfoque cognitivo en la comprensión lectora. Dicho modulo, repercutió en la toma de conciencia de los procesos mentales y en la comprensión lectora analítica, reflexiva e interpretativa al servicio de ulteriores aprendizajes académicos de mayor complejidad.

Otro estudio exploratorio, centró su interés en evaluar el efecto del tipo de tarea ajustada y ejecutada por el lector, es decir, su competencia lectora. Irigoyen, Jiménez y Acuña (2008), indican que «la evaluación de competencia lectora en correspondencia al comportamiento del sujeto frente a las condiciones de interacción (criterio de logro), apunta dos variables críticas al análisis de la misma: el contexto (ámbito) en donde el ajuste adquiere sentido y el tipo de requerimiento es expresado en la tarea a la cual debe atender».

Hay otro aspecto en los procesos de comprensión lectora que relaciona los procesos de aprendizaje con la comprensión de textos escritos en el ámbito educativo universitario. Desde allí, Fumero (2009), plantea como objetivo en su investigación, valorar la efectividad de un conjunto de estrategias didácticas que garantizarían el aprendizaje significativo y funcional de la comprensión de textos en los alumnos universitarios. De esta forma, logra concluir que las estrategias didácticas aplicadas y reflexionadas en su rediseño, permitieron la consolidación de los niveles de inferencia, evaluación y apreciación del texto. 
Dichas estrategias didácticas se discutieron y se concluyeron por medio de las siguientes categorías: Armando textos, comprendo textos a través de ilustraciones, construyo y aprendo, incluyendo todos los contenidos pedagógicos.

Más aún, los modelos de desarrollo de las estructuras de pensamiento mediante el análisis de la estructura de un texto, también han sido objeto de análisis en el aspecto de la comprensión lectora. Tolosa y Zerpa (2009), basados en el modelo de Kohlberg (1992), sobre el desarrollo moral, confirmaron dicha hipótesis en torno a la eficiencia de las estrategias de aprendizaje en el área de la lectura, específicamente acerca del mejoramiento de la comprensión a causa del aprendizaje de las estrategias que enmarcaron el programa de intervención; como lo es, la identificación de la macro-estructura del texto y las ideas principales.

Nuevamente, al indagar sobre los procesos de comprensión lectora en estudiantes de una institución privada, Martínez, Díaz y Rodríguez (2011), diseñaron y aplicaron un programa de andamiaje asistido para la comprensión lectora sobre los niveles de comprensión de textos científicos. En relación con los estudiantes, la investigación confirmó que la forma como se exige la lectura en el colegio, no guarda relación con lo esperado y contribuido en la universidad, ya que la calidad de los trabajos escritos en la fase inicial del programa, como resultado de la comprensión de un texto científico, presenta errores graves de interpretación en todos los niveles de comprensión. En cuanto a los profesores, encontraron que no hay relación entre lo que suelen exigir y lo que enseñan; al mismo tiempo, tampoco se integra la enseñanza de la lectura y la escritura en los diferentes cursos, lo cual puede funcionar como herramienta para ayudar a pensar los contenidos conceptuales y asegurar la enseñanza de las particularidades discursivas de cada campo.

Respecto a las estrategias cognitivas y meta-cognitivas que propician los docentes y a las que recurren los estudiantes para acercarse a las exigencias que requiere la comprensión lectora de los textos académicos; Cañón, Mancera y Ruiz (2011), a partir de los planteamientos de la etnografía educativa como disciplina, develan en su proyecto, que en pocas ocasiones un determinado grupo de estudiantes establece relaciones con la generación de estrategias para comprender lecturas. Al mismo tiempo, no se establece la pregunta por la pertinencia de cada estrategia, ni por su relación o coherencia con cada temática, actividad o forma de abordar la enseñanza; de la misma forma en la que tampoco se manifiesta un énfasis dado a la planeación y a la supervisión del propio proceso lector.

En último lugar, para ésta primera categoría «Comprensión de textos y estrategias cognitivas», vale la pena describir algunos aspectos centrales de la relación que establecen con la lectura los estudiantes universitarios. Rapetti y Vélez (2012), describen dicha relación, a partir del análisis de relatos de experiencias de lectura y escritos realizados por dos grupos de ingresantes a la universidad. Los 
resultados muestran vínculos entre posturas referentes y estéticas expresados en los relatos realizados a partir de: lo que puede ser evocado por los lectores y lo que puede ser evocado por la lectura. Por otra parte, los relatos analizados muestran otras maneras de leer, guiadas por posturas predominantemente estéticas, en las que el contexto y el texto activan otros «guiones» de lectura que culturalmente «permiten» expresar el compromiso del Self.

\section{Comprensión de textos virtuales}

Este segundo apartado, reconoce que el mundo virtual hace parte indiscutible de las modalidades educativas. Se construye sin ánimo de arrojar prejuicios, sino con el fin de entender sus configuraciones respecto a los procesos de lectura. El primer interés, indaga la correspondencia entre el rendimiento del alumno con el modo de navegar y con los estilos de aprendizaje. Frente a este tema, Gaspar (2008), examinó el hecho de abrir nuevas vías para potenciar el aprendizaje intercultural y trabajar al mismo tiempo la comprensión lectora, determinando así que los alumnos no leen de igual forma en pantalla, que en texto, puesto que los alumnos reconocen prestar más atención a las palabras clave cuando trabajaban en pantalla, que cuando leen en formato papel, aventurándose a realizar presuposiciones sobre el texto.

Una muestra de este tipo de prácticas, se manifiesta en la dinamización de las bibliotecas sobre la comprensión lectora. La experiencia de García (2012), consistió en entregar el libro, tanto en papel como en formato digital, para ser leído en la tableta iPad de Apple. Para la investigación se utilizaron diversas herramientas como los cuestionarios, entrevistas y grupos de discusión (focus group). Esto le permitió encontrar: «En la fase semidirigida, los lectores guiados (los del grupo experimental que habían participado en las actividades de las bibliotecas) aportan más detalles sobre el personaje principal, mencionan más personajes, captan mucho más sobre el contexto musical y con rotundidad ofrecen más detalles sobre la trayectoria de los Beatles».

\section{El maestro como mediador en la experiencia de comprensión}

Esta categoría, parte por identificar qué actividades permiten la enseñanza de estrategias de comprensión lectora. Para responder a ello, Paradiso (1996), identifica algunas estrategias, tales como: descartar, seleccionar, subtitular, identificar proposición, temática, jerarquizar (macrorreglas), resumir, representar la superestructura, construir un mapa cognitivo, entre otros. Su aplicación en la respectiva investigación permitió identificar el rol del docente; no como expositor de conocimientos, sino como mediador o planificador reflexivo, primero, con el fin de hacer emerger los conocimientos previos y segundo, para motivar a los estudiantes e inducir su auto-actividad. Por lo demás, la definición de estas estrategias, caracteriza a los buenos lectores, como aquellos que toman decisiones acerca de qué estrategia usar, cuándo usarla y cómo adaptarla a un texto en particular. Además, los buenos lectores usan una conciencia metacognitiva; es decir, su razonamiento y pensamiento crítico, mientras construyen 
y reconstruyen los significados sucesivos del texto, adaptándose a diferentes tipos de textos y diferentes propósitos.

Para ubicar la comprensión de textos en el área universitario Rincón, Narváez y Roldan (2004), aportan específicamente acerca del qué y cómo se está haciendo. De esta forma, ponen en discusión algunos de los hallazgos obtenidos en la investigación que venían realizando: Interactividad y enseñanza de la comprensión de textos escritos académicos en cursos presenciales y no presenciales en el ámbito universitario. La investigación identificó en el nivel de microanálisis del grupo no presencial, contradicciones entre los contenidos y los procedimientos que se encuentran en el modulo. Para las autoras, hay mayor articulación profesor-alumno en el curso presencial; por tanto la desvinculación en el curso no presencial, requiere el acompañamiento del tutor para que los alumnos no se sientan solos y deserten; puesto que, la relación e intercambios entre profesor- alumno solo se está evidenciando en responder preguntas y plantear soluciones, sin permitir un ejercicio de verbalización e interiorización de las dificultades del estudiante al construir un texto.

Con el fin de ahondar acerca de la participación docente en la compresión lectora de estudiantes universitarios, Ortega y Andrade (2007), presentan algunas conclusiones de la participación activa del docente en la inducción de un modelo estratégico para la comprensión de la lectura en estudiantes universitarios. Frente a las debilidades que presentan: los estudiantes requieren reforzar sus estrategias de aprendizaje de adquisición, organización (elaboración de esquemas), recuperación de la información (autopreguntas y resúmenes) y de aplicación de la información (solución de problemas, planteamientos de ejemplos de la vida práctica), dando uso a la lectura como un medio de aprendizaje.

Ahora bien, Makuc (2008), logra identificar cuatro teorías implícitas de los profesores acerca de la comprensión de textos: teoría lineal, teoría cognitiva, teoría interactiva y teoría transaccional. Estas teorías constituyen un marco de referencia para interpretar y categorizar las teorías implícitas de los docentes de acuerdo a tres sub-dominios fundamentales en el ámbito de la comprensión: noción de comprensión, noción de lector y noción de texto. Este estudio muestra que el profesor en el contexto de la enseñanza ajusta o modifica su teoría de la comprensión a las características del contexto y a las demandas a las que se ve enfrentado.

En el último hallazgo de este tercer subgrupo, se tensiona como objeto de formación docente, la enseñanza de la lectura académica. En relación con este proceso, Narváez y Cadena (2009), por medio de un estudio previo sobre tareas de lectura en cursos profesionales promueven: primero, la importancia de abordar la enseñanza de la lectura académica en la educación superior y segundo, un ejercicio dialectico sobre algunos de los mitos relacionados con la lectura en el ámbito universitario. Lo anterior permitió concluir que para 
incidir sobre el ejercicio del derecho a una educación superior de calidad y con mejores procesos en lectura en los estudiantes en formación, se debe proponer a las direcciones universitarias la conformación de equipos colegiados e interdisciplinarios orientados con profesores especializados en el campo de la enseñanza de la lectura académica, para dar lugar al intercambio de experticias y reflexiones sobre sus prácticas.

\section{Lectura y escritura en la universidad}

En este cuarto sub-grupo, se hace oportuno destacar un curso-taller de lectura y escritura impulsado por Castañeda y Henao (1999). En su investigación, el principal objetivo fue establecer la relación entre lectura, escritura, estrato social y rendimiento académico de los estudiantes. En cada sesión de clase, el estudiante debía leer y escribir; en la clase siguiente se evaluaban, se comentaban los textos y se analizaban las estrategias que se aplicaron tanto en el proceso lector como en el de escritura; finalmente, se hacían sugerencias para mejorar estas prácticas.

Al identificar que «la creatividad» cumple una función importante en los procesos de lectura y escritura; Rolando, Bidiña, Zerillo, Pidoto, Espelta, Callegaro y Motta (2002), discuten el hecho de que los estudiantes se apropien de los modelos formales con escasa creatividad, puesto que, no pueden realizar las operaciones necesarias para que esas estructuras sean textualmente significativas. Además, repiten formas tanto en nivel estructural como léxico y estilístico, evitando el punto crítico y personal. De ahí que la llamada «alfabetización académica», requiera un entrenamiento especial en lectura y escritura que la universidad exige pero no enseña.

Para indagar acerca del mejoramiento de los procesos de comprensión de textos académicos en el ámbito universitario; Perilla, Rincón, Gil y Salas (2004), realizan una revisión de elementos conceptuales y metodológicos que orientan el diseño de una prueba de comprensión de lectura aplicada a unos estudiantes de pregrado ingresantes. La investigación demostró que los problemas de comprensión se ubican en el nivel de lectura inferencial y están referidos a los modos de organización de orden explicativo y argumentativo. Esto les permitió recomendar la implementación de seminarios con otros profesores y directores de programas de estudio; así como, la realización de talleres de lectura y escritura para la comunidad universitaria, el diseño de cursos de comprensión y producción de textos académicos para propósitos disciplinarios específicos y la publicación de materiales didácticos que potencien la cultura de lectura y escritura como vehículo de apropiación y construcción de conocimiento.

Así pues, a partir de la preocupación que presentan algunos docentes de lengua extrajera acerca de las diversas dificultades de los estudiantes universitarios que corresponden al manejo del idioma castellano; Arrieta de Meza y Meza (2005), señalan que algunas de estas dificultades corresponden a: incapacidad para identificar ideas principales expuestas en un texto; imposibilidad de captar 
la intención del escritor; y dificultad en la redacción incoherente de resúmenes de los textos objeto de lectura. Luego de su respectiva intervención, logran destacar tres factores que intervienen en la redacción errónea de los estudiantes universitarios: la falta de definición de un plan de trabajo antes de comenzar una redacción; obtener poca información, debido al poco interés hacia la lectura; y las incorrecciones idiomáticas. Al mismo tiempo, los investigadores señalan que los problemas tratados en este trabajo corresponden no sólo a los profesores de lenguas, sino también a los de otras asignaturas, ya que influyen directamente en el manejo de los contenidos teóricos de disciplinas, tales como: biología, historia, pedagogía, sociología, matemática o geografía.

Para profundizar en el aspecto de construcción y reformulación textual, la caracterización de los tres tipos de reformulación: resuntiva, comprensiva y productiva, realizada por Piacente y Tittarelli (2006), les permitió observar dificultades de puntuación, de cohesión textual, y omisiones semánticas relativas a la extracción de las partes principales del texto. Cuando los autores se interrogan sobre la naturaleza de dichas dificultades, ligadas a problemas de producción y a la enseñanza universitaria en el tratamiento textual; llama la atención el desconocimiento de los alumnos sobre la organización retórica de un resumen o sobre los alcances de tareas de reemplazo de segmentos de texto. Esta circunstancia, les obligó a pensar de qué manera los estudiantes realizan sus propios resúmenes, cuando interactúan con textos académicos, puesto que la producción textual constituye una de las medidas indirectas de la comprensión y exige lo comprendido a través de reformulaciones tales como: la elaboración de resúmenes y las paráfrasis de segmentos de textos.

Hasta aquí, se comprende el señalamiento que realizan algunos profesores, acerca de la necesidad de ciertos niveles de lectura con los que el alumno debe contar, cuando decide ingresar a la Universidad. Al respecto, Rodríguez (2007), resalta en su investigación «las habilidades de pensamiento», como una de las características más significativas que deben desarrollar los alumnos. Dicha habilidad, conforma el «piso cognitivo» que los docentes esperan de los estudiantes que ingresan al nivel universitario. No obstante, dicho supuesto contrasta con la recurrente identificación de «falencias o dificultades» en alumnos, que lejos de lograr un aprendizaje autónomo y comprensivo, demandan mayores cuotas de dependencia del docente para comprender el contenido académico. Así pues, «La falta de lectura» es considerada la responsable de mayor peso en las dificultades.

Lo cierto es que las conclusiones de la mayoría de investigaciones, aboga por una comprensión crítica que desarrolle procesos de lectura enfocados a problematizar ideas y hechos, así como, descubrir intenciones e ideologías y adoptar puntos de vista. Es el caso de la propuesta didáctica de Serrano (2008), basada en el estudio y reflexión teórica sobre la lectura crítica en el contexto universitario; teniendo en cuenta que uno de los objetivos de la educación 
universitaria, ha de ser el desarrollo del pensamiento crítico de los estudiantes para acceder al conocimiento; por lo tanto para Serrano, en la universidad es preciso entender que aprender a leer y desarrollarse como lector se va logrando progresivamente, de tal modo que, cuando los estudiantes ingresan a estudios superiores aún necesitan continuar desarrollando competencias en lengua escrita para interpretar teorías y conceptos abstractos, a través de procesos y fenómenos, puesto que, en la educación superior se impone la necesidad de formar un lector crítico y multidisciplinario.

Las dos anteriores investigaciones, argumentan el hecho de concebir los procesos de lectura aunados a los procesos de escritura. De igual forma, Uribe y Martínez (2008), realizan una revisión acerca de las Prácticas de Lectura y Escritura Académicas en la Universidad Colombiana. En dicho artículo, se presenta el análisis de algunas prácticas de lectura y escritura académicas en la universidad colombiana, conocidas por diversos medios, para aportar a la reflexión y el diálogo académico entre profesionales de la educación, a partir de experiencias en campos problemáticos específicos. Entre las conclusiones se destaca el considerar que la escritura académica se exige pero no se enseña, posiblemente, porque se le supone como una habilidad general, aprendida en niveles educativos previos y transferibles a cualquier situación.

En cuanto a la propuesta interdisciplinar que implica relacionar los procesos de lectura y escritura, Arnáez (2009), expone la acción metodológica de un trabajo de campo en el área de matemática; en el cual, se aplicaron y valoraron cuatro cuestionarios; cuyos resultados, sirven de base en el diseño de una propuesta orientada por la secuencia didáctica. La conclusión fundamental de este trabajo fue: la lectura y la escritura deben ser tratadas de manera interdisciplinar y como compromiso de todos, puesto que, existen necesidades específicas sobre textos académicos especializados para las que debe aplicarse un proyecto de lectura y escritura relacionado con el currículo y las disciplinas.

Más aun, el interés por la lectura y la escritura académica en la universidad, desde el punto de Narváez y Chois (2012), demuestra que en Colombia la lectura y la escritura coexisten con algunos de los fenómenos académicos denominados procedimientos de iniciación, específicamente con las prácticas pedagógicas relacionadas con la enseñanza en el aula. Una vez los resultados de su investigación ratifican que las prácticas de enseñanza primero, son parte de la cultura académica relacionada con la lectura y la escritura y segundo, son determinantes en la formación de las generaciones académicas y profesionales más jóvenes; las autoras, logran confirmar que en Colombia se han ocupado de estudiar las dificultades que traen los estudiantes desde la escolaridad previa y eso hace que los mismos estudiantes perciban que se lee y se escribe en la universidad para tener una formación especializada en el campo de las disciplinas y las profesiones, reconociendo que lo que sucede con la lectura y la escritura en la universidad es distinto y no guarda relación alguna con lo que viven en la escolaridad previa. 
Sin embargo, acomete la siguiente inquietud: ¿En qué se diferencian las prácticas de lectura y escritura de la universidad y las de la escuela secundaria? En su autoría, Fernández y Carlino (2010), exploran los desafíos que enfrentan los ingresantes a la universidad y logran abordar diferencias sustantivas entre ambos niveles educativos y el reto que implican. Así pues, la investigación destaca la necesidad de que la educación secundaria vuelva a pensar las tareas de lectura y escritura a las que suele dar lugar, puesto que, desde el punto de vista de estos estudiantes que ingresan a los primeros periodos de la universidad, muchas de ellas pueden hacerse sin comprender los textos leídos.

Desde esta perspectiva, Rincón y Gil (2010), siguen el objetivo de describir, caracterizar, analizar e interpretar las prácticas de lectura y escritura dominantes en la universidad colombiana. Una de las tendencias dominantes, relacionadas de manera general con actividades pragmáticas o instrumentales; señala que a los estudiantes se les solicita leer y escribir para responder sobre todo a las exigencias académicas de los planes de estudio y menos a otras prácticas de corte investigativo, de divulgación y proyección de la formación universitaria y ciudadana en general. Los autores encontraron que no se presenta una articulación efectiva entre las declaraciones, consensos teóricos, definiciones institucionales de orden curricular en general desde las unidades académicas.

De esta forma, la información obtenida le permitió a Rincón y Gil (2010), dar paso a la elaboración de: El estado de tendencias en las prácticas de lectura y escritura académicas en la Universidad del Valle y abordando la problemática de la lectura y la escritura en la universidad colombiana desde las apuestas pedagógicas y didácticas que en ella hoy se concretan. El trabajo fue adelantado por un grupo interuniversitario que, con el apoyo de Colciencias y cada una de las 17 universidades participantes, adelantó la investigación denominada ¿Para qué se lee y se escribe en la universidad colombiana? Un aporte a la consolidación de la cultura académica. Así pues, se puede constatar la presencia de prácticas fuertemente ancladas en la tradición pedagógica heteroestructurante, que siguen mediando la formación de los estudiantes, es decir, las orientaciones didácticas y pedagógicas, asociadas a concepciones instrumentales de lo que significa leer y escribir en el mundo académico. Para los autores, es preocupante aún la comprobación de que estos resultados son los que dominan en el conjunto de las universidades en la que esta investigación también se realiza.

Conjuntamente el ejercicio de Parra (2012), derivado de la investigación general «¿Para qué se lee y se escribe en la universidad colombiana?», demanda un acercamiento a la noción de cultura académica, como lugar conceptual y estratégico que desde su dimensión pedagógica, le da potencia a una mirada sobre las prácticas de lectura y escritura en la universidad. Sugiere algunas preguntas a la didáctica universitaria que precisan una concepción amplia de lo pedagógico, lo didáctico y de las prácticas de lectura y escritura, puesto que, estos conceptos serán los lentes que permitirán potenciar una mirada didáctica, 
resistente a discursos centrados exclusivamente en lo cognitivo, normativo, técnico, administrativo e instrumental. Según Parra, discurrir sobre «la cultura académica», implica reconocer que en las prácticas de lectura y escritura se materializan relaciones de saber-poder-verdad, como podría evidenciarse en las tensiones de toda índole que transitan en la vida y formación universitaria en Colombia, tales como: estudio vs trabajo, práctica pedagógica vs práctica científica, formación vs instrucción, producto vs proceso.

A las apreciaciones de la investigación «¿Para qué se lee y se escribe en la Universidad Colombiana?», se suma el artículo realizado por García (2011), quien considera que el profesor, usualmente, expone las temáticas mientras los estudiantes toman nota del objeto sobre el que se discurre. Según García, esta tendencia generalizada mostraría, por un lado, que el «discurso oral» del profesor sigue siendo central para los estudiantes a la hora de comprender y apropiarse de ese saber comunicado; de otra parte, esta práctica escritural de «reproducción fiel del discurso docente» se vincula con aquello que será evaluado. Esto coincide justamente con el propósito de la lectura frecuente tanto en Educación, como en las demás áreas de Unesco: «leer para responder preguntas al profesor». Además, le permite corroborar que las prácticas de lectura y escritura más relevantes para los estudiantes están directamente ligadas a las áreas específicas del saber disciplinar. Es decir, una vez los estudiantes han adquirido cierto dominio temático y conceptual de aquello para lo cual se forman; leer y escribir adquiere mayor significado.

A partir de los artículos encontrados, se puede cuestionar: ¿Qué se ha planteado como estrategia para mejorar los niveles de comprensión de textos académicos? $\mathrm{Su}$ respuesta se dirige hacia los aportes de tres investigaciones. La primera, realizada por Gonzales, Castañeda, Maytorena y Gonzales (2008), expone un análisis factorial confirmatorio que permitió construir un modelo estructural de comprensión de lectura integrado por dos variables latentes de primer orden (Niveles fácil y difícil de evaluación).

La segunda investigación, realizada por Moyano (2010), presenta un programa para el desarrollo de habilidades de lectura y escritura a lo largo de la carrera universitaria basado en la teoría de género y registro. Además, exige el compromiso de actores de diferente jerarquía institucional y diferentes especialidades disciplinares, además de los docentes en ciencias del lenguaje. La tercera investigación, (último lugar para el cuarto sub-grupo «Lectura y escritura en la universidad», dirigido a maestros) efectuada por Irrazabal (2010), buscó evaluar la influencia del conocimiento previo en la comprensión de textos expositivos generales y específicos. Estos resultados apoyarían la hipótesis según la cual el conocimiento previo sería el principal factor explicativo de la diferencia de rendimiento entre ambos grupos. 


\section{Lectura y escritura en educación virtual y a distancia}

El procesamiento cognitivo y comprensión de textos en formato hipermedial, se convierte en el fundamento de la quinta categoría. Según Henao (2002), el texto electrónico y los materiales multimediales están generando transformaciones en el oficio de escribir y en el acto de leer; en la producción, edición y distribución de diversas publicaciones; en la relación autor-lector y en la promoción de la lectoescritura. De esta forma, las reflexiones de la investigación giran alrededor del interés por la relación y mutua influencia entre las «nuevas tecnologías» y el aprendizaje en torno a: el dominio del vocabulario, el uso del diccionario, el análisis contextual, la comprensión lectora, los mapas semánticos, la identificación de ideas principales y de detalles, la habilidad para resumir y para recordar la información consignada en textos hipermediales e impresos.

Para ahondar en los fenómenos de la «lectura y escritura» en medios electrónicos, Argüello (2012), se centra en las migraciones digitales de lectura y escritura en estudiantes universitarios, con el fin de analizar la forma como los estudiantes migran digitalmente, (dejan un lugar y se dirigen a otro) al crear blogs, realizar búsquedas en google, seleccionar fuentes documentales y citar. Así pues, la Lectura y la escritura son concebidas como un asunto de interconexión e intertextualidad, un paso de cultura analógica a una cultura digital, ya que el auge de internet ha modificado la manera de realizar consultas de fuentes documentales y bibliográficas para la redacción de trabajos universitarios.

\section{Escritura}

Conforma el sexto subgrupo, la investigación realizada por Novo, Tenca y Escalarea (2008), quienes consideran que la escritura académica como práctica, propicia una transformación en la concepción epistemológica de los estudiantes. De esta forma, asumir la voz en la palabra escrita implica hacerse cargo de lo dicho, contraer un compromiso y dicha responsabilidad se juega al menos en dos sentidos que confluyen en la escritura: compromiso con la propia voz en cuanto principio de una coherencia, de una identidad que se escribe y se expone-impone a otro, y con las voces de los enunciadores que el propio texto evoca y somete a un diálogo armónico, a una disputa o a una discusión sin cuartel.

Acerca de la escritura académica en estudiantes universitarios, Bocca y Vasconcelo (2009), presentan algunas reflexiones. Si los docentes son conscientes de que el alumno universitario debe desarrollar estrategias especiales para encarar sus prácticas lectoras, también han de reconocer, que estos deben adquirir habilidades para la producción de textos académicos; puesto que, las representaciones de la escritura y de la lectura encierran la idea de composición y figura del escritor, como medio para expresar sentimientos, sujetos a la inspiración y la creatividad; lo cual, anularía la concepción de la escritura como un proceso cognitivo y práctica social. 


\section{Escritura, dirigido a maestros}

Este séptimo apartado, inicia por la problemática que mas inhibe al alumno, cuando se dispone a escribir para otro y a interrelacionarse con otros. Esta problemática, se refiere al destinatario en los planos de la escritura y la realidad. Desde allí, Sosa y Loss (2007), observan en los alumnos, una falta de identificación de los destinatarios reales, demostrando que solo identifican al profesor como destinatario y crítico de sus escritos. Esto evidencia, que los alumnos salen de la universidad sin la adquisición conceptualizada del modelo de escritura «para transformar el conocimiento».

Ahora bien, frente a la escritura académica en la formación del docente universitario Patiño (2010), en su reflexión más destacada, expone que la tarea de escribir representa para el docente una actividad que implica altos niveles de dedicación, que no son posibles de cumplir debido a otras ocupaciones. Del mismo modo, advierte la idea de que la escritura no ha sido considerada una actividad relevante dentro de la labor docente o académica; primero, debido a que es desplazada por otras como la planeación de las clases y la evaluación del aprendizaje de los estudiantes; y segundo, porque posiblemente no merece la dedicación y el tiempo requerido.

Finalmente, Gutiérrez, Romero, Flórez y Saldarriaga (2011), postulan estudios avanzados acerca de una actividad cognitiva y su repercusión en la cognición humana, específicamente en la lectura de ciertos tipos de texto; por ejemplo en el narrativo, científico y expositivo. La investigación arroja una serie de postulados a tener en cuenta para el planteamiento de las estrategias, aun no tratados, en las practicas de docentes y estudiantes universitarios: no saben leer y escribir, no poseen hábito lector, no comprenden porque no leen», y esto propicia «falta de lectura».

\section{Discusión}

Los artículos reseñados, procuran una visión global sobre el campo estudio de interés. A su vez, posibilitan relaciones entre la comprensión y producción de textos académicos en educación superior, tanto nivel presencial como virtual y a distancia; para definir cuáles han sido desarrollados y en cuáles se considera necesario ampliar su indagación. Teniendo en cuenta la necesidad de concienciar a maestros y estudiantes sobre la importancia de la lectura y la escritura, como formas de acceso al conocimiento y cultura académica predominante en las universidades; los siguientes, son análisis realizados por la congruencia de dichas publicaciones y aportes que entre sí pueden llegar a realizar.

- Algunas de las dificultades identificadas de los estudiantes universitarios se reflejan en el manejo del idioma castellano, en la comprensión lectora y la redacción correcta o adecuada al nivel académico universitario. El problema se 
evidencia en la incapacidad del individuo para identificar las ideas principales expuestas en un texto, en la imposibilidad de captar la intención del escritor y en la redacción incoherente de resúmenes de los textos objeto de lectura; problemas que deben ser tratados por los profesores de todas las asignaturas, ya que en el manejo de los contenidos teóricos, influye la disciplina que se busca desarrollar.

- La mayoría de las investigaciones, plantean que los estudiantes a la hora de iniciar sus estudios universitarios carecen de: estrategias de estudio, conocimientos generales, conciencia de los procesos intelectuales, identidad en el desempeño académico, análisis de las tareas académicas y del uso de textos durante las clases de distintas asignaturas, entre otros factores, que conforman el currículo de la secundaria. Por tanto se considera como resolución a las dificultades en comprensión y producción de textos, la efectividad de acciones pedagógicas, que dinamicen procesos metacognitivos.

\section{Acerca de los docentes}

- Como fruto de la reflexión, surge la necesidad de desarrollar proyectos que involucren de manera protagónica a todos los actores del proceso de formación, tanto estudiantes como maestros, en la investigación de los procesos de lectura y escritura. Con el fin de optimizar dichos procesos en los primeros periodos de educación superior, se considera fundamental diseñar o retomar la experiencia de la Universidad del Valle, con una prueba de ingreso que evalúe los niveles cognitivos y los procesos de comprensión y producción de textos con los que cuenta el aspirante, para retomar esta información en un proceso de seguimiento que defina un plan de acompañamiento en el proceso, con el apoyo de estrategias pedagógicas de comprensión y producción de textos que permitan resolver las dificultades identificadas.

- La mayoría de los estudiantes universitarios presentan problemas en los niveles básicos y avanzados del desarrollo cognitivo, implícitos en el proceso de comprensión de textos, especialmente en la representación mental de textos científicos; por tanto, el compromiso implica desarrollar diferentes estrategias tanto en el campo del desarrollo cognitivo desde diferentes asignaturas o espacios académicos.

\section{Textos en la red}

- El enfoque que tiende a profundizar el fenómeno comunicacional de lectura y escritura en el horizonte de los nuevos medios electrónicos, deja ver las migraciones digitales del lector y escritor universitario que ocurren al organizar una página, debido a la influencia de Google en la búsqueda y selección de fuentes, documentales y bibliografías, así como en la forma de incorporar citas en los textos. Ello sucede por el auge de internet, el cual ha modificado la manera de realizar consultas de fuentes documentales y bibliográficas para la redacción de trabajos universitarios. 


\section{Formación para la lectura crítica}

- En algunas investigaciones se resalta la función de comprensión crítica, inmersa en la educación universitaria. Su desarrollo requiere incrementar en los estudiantes capacidad de lectura crítica, comprensión y reflexión para problematizar las ideas y los hechos, descubrir intenciones e ideologías y adoptar puntos de vista. Además, si aprender a leer se convierte en un proceso, entonces, no se puede aspirar en los estudiantes que ingresan a estudios superiores, un nivel final; por el contrario, se necesita continuar desarrollando competencias en lengua escrita para interpretar teorías, conceptos abstractos y así finalmente, ser capaces de explicar fenómenos.

- Interesa examinar sobre cómo se podría investigar la comprensión de textos en el área virtual y a distancia, siguiendo los planteamientos que proponen las investigaciones del entorno presencial. Es importante identificar en la modalidad virtual cómo se diseñan las didácticas que están mediando las prácticas académicas de lectura y escritura en el marco de las asignaturas, comprender las interacciones entre los participantes y los documentos que circulan para ser leídos y sobre los cuales se producen escritos.

- En la modalidad virtual contribuye la idea de mejorar los procesos de lectura y escritura, a través de una adecuada acción pedagógica, proveniente de pruebas que pueden identificar tanto los niveles cognitivos con que cuenta el estudiante al ingresar a la educación superior, como los procesos de compresión de lectura y producción de textos para la vida académica.

Estrategias que se han desarrollado para mejorar los niveles de comprensión de textos

- El desarrollo y aplicación de estrategias de meta-cognición para la comprensión y producción de textos permite al estudiante mejorar los procesos cognitivos implícitos en la lectura y la escritura. Dichas estrategias, son muy útiles en la planificación de mediaciones pedagógicas para propiciar la producción de significados y encontrar sentido al uso de ciertas técnicas, tales como: principios, reglas y estrategias.

- Los estudiantes requieren dominar los recursos lingüísticos y discursivos de su disciplina, por tanto es necesario que el proceso de formación del profesional motive a apropiarse de la estructura de los discursos, para lograr contextualizar los referentes de los estudiantes con las características discursivas esenciales en apropiación y construcción de conocimiento de cada campo del saber.

- En el proceso de producción de textos, es fundamental reconocer y potenciar la propia voz de los estudiantes, propiciar su afirmación y expresión a través de la escritura con coherencia, con el fin de permitirse un encuentro y configurar su identidad a la hora de escribir. 


\section{Reflexiones}

En en los proceso de formación «comprensión y producción de textos académicos», se requiere establecer vínculos de significación entre lo que el estudiante ha construido en sus experiencias previas, para suscitar en ellos una resignificación de este proceso a partir de la vida universitaria.

\section{Comprensión de textos en ambientes virtuales}

Teniendo en cuenta los resultados de la investigación de Gaspar (2008), según la cual, los estudiantes no leen igual en pantalla que en texto y que la pantalla les permite reconocer con mayor atención las palabras clave para seleccionar los textos de su interés; se requieren propuestas pedagógicas comprometidas con la construcción de los procesos implícitos en el proceso de comprensión de lectura desde una perspectiva crítica, para potenciar el aprendizaje intercultural utilizando las ventajas de los medios electrónicos, las redes y las bases de datos a las cuales puede acceder el estudiante.

Para continuar este proceso reflexivo se propone realizar una investigación en el contexto de la Universidad Nacional Abierta y a Distancia, que se anime a diagnosticar problemáticas y plantear estrategias en educación virtual y a distancia. Una vez se reconoce que la mayoría de las investigaciones en procesos de lectura y escritura están orientadas hacia el ámbito educativo presencial, puede tenerse en cuenta sus planteamientos e indagar su aplicación en la educación virtual y a distancia. De ahí que, los docentes, pedagogos, diseñadores de cursos y de software, deben manejar conocimientos en recursos hipermediales y modelos que propicien a través del blended-learning y movil-learning, la posibilidad de acceder a textos académicos en sus prácticas comunicativas.

\section{El docente como mediador en la experiencia de comprensión}

El rol del docente se trasforma en los procesos de formación en lectura y escritura; ya no es visto como un expositor de conocimientos sino como un mediador frente a los textos, las experiencias y las diversas culturas. Esta relación, busca que los estudiantes logren hacer análisis sobre el texto a través de un pensamiento crítico que permita construir y reconstruir los significados del texto, capaces de adaptarse a diferentes tipos de textos y narrativas. El docente mediador, solo acompaña el proceso de aprendizaje por medio de estrategias para el aprendizaje, adquisición y organización de significados, que implican el uso de esquemas cognitivos con el fin de desarrollar en el estudiante la capacidad de recuperar el sentido del texto, a través de resúmenes, reseñas y tematizaciones.

\section{La institucionalidad de los procesos de comprensión y producción de textos}

Teniendo en cuenta la profunda relevancia que tienen los procesos de comprensión y producción de textos para superar las inequidades o debilidades de los estudiantes al ingresar en educación superior, es necesario que las políticas de las Instituciones de Educación Superior se organicen y reflejen en sus apuestas 
macrocurriculares, apoyo y aporte en la formación integral, el acceso a las diversas culturas, la generación de condiciones y de posibilidades para producir conocimiento con artículos científicos.

\section{Referencias bibliográficas}

Arguello, G. 2012. Migraciones digitales de lectura y escritura en estudiantes universitarios. Revista de Universidad y Sociedad de Conocimiento 9(1): 5-21. Disponible en: http://redalyc.uaemex.mx/src/inicio/ArtPdfRed.jsp?iCve=78023415002 [Fecha de consulta: junio 29 de 2012].

Arnáez, P. 2009. Leer y escribir en la universidad: una propuesta interdisciplinar. Enunciación 13: 7-19. Disponible en: http://200.69.103.48/comunidad/grupos/ lenidencultura/revista/enunciaci\%F3n\%2013/3.\%20Leer\%20y\%20escribir\%20 (Arnaez)\%20enunciacion13.CV01.pdf [Fecha de consulta: junio 30 de 2012].

Arrieta, B. \& Meza, R. C. 2005. La comprensión lectora y la redacción en estudiantes universitarios. Revista Iberoamericana de Educación 35 (2). Disponible en: http:/l www.rieoei.org/deloslectores/825Barrieta.PDF [Fecha de consulta: agosto 1 de 2012].

Bocca, A. \& Vasconcelo, N. 2009. Algunas reflexiones acerca de las prácticas y representaciones sociales en estudiantes universitarios: la escritura académica. Enunciación 13: 20-27. http://200.69.103.48/comunidad/grupos/lenidencultura/ revista/enunciaci\%F3n\%2013/4.\%20Algunas\%20reflexiones\%20(Bocca-Vasconcelo) enunciacion13.CV01.pdf [Fecha de consulta: junio 28 de 2012].

Brugueras, M., Díaz, G., Díaz A., \& Valdés, M. 2008. El artículo de revisión. Revista Cubana de Salud Pública. 34: 1-11. Disponible en: http://redalyc.uaemex.mx/src/inicio/ ArtPdfRed.jsp?iCve=21419854011 [Fecha de consulta: julio 6 de 2012].

Cañón, L., Mancera, J. \& Ruiz N. 2011. La Lectura de los textos académicos en la formación universitaria de docentes: entre supuestos y estrategias. Pedagogía y Saberes 33. Disponible en: http://revistas.pedagogica.edu.co/index.php/PYS/article/view/760 [Fecha de consulta: julio 10 de 2012].

Castañeda, L. \& Henao, J. 1999. La lectura en la Universidad de Antioquía: Informe preliminar. Signos 45-46: 83-11. Disponible en: http://www.scielo.cl/scielo. php?script=sci_arttext\&pid=S0718-09341999000100010 [Fecha de consulta: julio 6 de 2012].

Echevarría, M. 2006. ¿Enseñar a leer en la universidad? Una intervención para mejorar la comprensión de textos complejos al comienzo de la educación superior. Psicodidáctica 2: 169-188. Disponible en: http://www.ehu.es/ojs/index.php/psicodidactica/article/ view/198 [Fecha de consulta: julio 10 de 2012]. 
Escudero I. \& León, J. 2007. Procesos inferenciales en la comprensión del discurso escrito. Influencia de la estructura del texto en los procesos de comprensión. Signos 64: 311-336. Disponible en: http://www.scielo.cl/scielo.php?pid=S071809342007000200003\&script=sci_arttext [Fecha de consulta: junio 10 de 2012].

Fernández, G. \& Carlino, P. 2010. ¿En qué se diferencian Las prácticas de lectura y escritura de la universidad y las de la escuela secundaria? Lectura y Vida 3: 7-19. http:// www.lecturayvida.fahce.unlp.edu.ar/numeros/a31n3/31_03_Fernandez.pdf [Fecha de consulta: junio 29 de 2012]

Fumero, F. 2009. Estrategias didácticas para la comprensión de textos. Una propuesta de investigación- acción participativa en el aula. Investigación y Postgrado 1 (4673). Disponible en: http://redalyc.uaemex.mx/pdf/658/65815763003.pdf [Fecha de consulta: julio 9 de 2012].

García Vera, N. 2011. Cultura académica y prácticas de lectura y escritura, a propósito de la formación universitaria de docentes. Pedagogía y Saberes 35: 117-139. Disponible en: http://revistas.pedagogica.edu.co/index.php/PYS/article/view/951/965 [Fecha de consulta: julio 11 de 2012].

García, R. 2012. La dinamización en las bibliotecas tiene efectos relevantes sobre la comprensión lectora. Lectura Lab. El laboratorio de la lectura de la FGSR (Fundación German Sánchez Ruipérez). Disponible en: http://www.lecturalab.org/story. php?id=2180 [Fecha de consulta: julio 15 de 2012].

Gaspar, M. 2008. Estrategias de comprensión de textos virtuales de temática intercultural. Magazín 18: 46-51. Disponible en: http://dialnet.unirioja.es/servlet/ articulo? codigo $=2879190$ [Fecha de consulta: agosto 20 de 2012].

Gonzales, L., Castañeda, D., Maytorena, M., Gonzales, N. 2008. Comprensión de textos en estudiantes universitarios: dos contextos de recuperación de información. Revista de la Educación Superior 146: 41-45. Disponible en: http://www.scielo.org.mx/scielo. php?pid=S0185-27602008000200003\&script=sci_arttext [Fecha de consulta: julio 10 de 2012]

Gutiérrez, M. , Romero, J., Flórez, R. \& Saldarriaga, C. 2011. Enseñar a escribir en la universidad: saberes y prácticas de docentes y estudiantes universitarios. Magis 4 (7): 137-168. Disponible en: http://revistas.javeriana.edu.co/index.php/MAGIS/article/ view/3561/2675 [Fecha de consulta: junio 27 de 2012].

Henao, O.2002. Procesamiento cognitivo y comprensión de textos en formato hipermedial. Medellín: Editorial Universidad de Antioquia.

Irigoyen, J., Jiménez, M., Acuña, K. 2008. Análisis de la competencia lectora en estudiantes universitarios. Boletín Electrónico de Investigación de la Asociación Oaxaqueña de Psicología, 1: 84-96. Disponible en: http://www.conductitlan.net/52_ competencia_lectora.pdf [Fecha de consulta: agosto 15 de 2012]. 
Irrazabal, N. 2010. La comprensión de textos expositivos en estudiantes universitarios: la función del conocimiento previo. Revista de Psicología 12: 7-21. Disponible en: http:// bibliotecadigital.uca.edu.ar/repositorio/revistas/comprension-textos-expositivosestudiantes-universitarios.pdf [Fecha de consulta: junio 29 de 2012].

Lucero, S., Vitale, M., Iturralde, M., Valente, G. \& Mazzitelli, C. 2006. ¿Todos los estudiantes que llegan a la universidad han alcanzado el nivel de operaciones formales? Un estudio comparativo entre la frm - utn y Facultad de Ingeniería en Uncuyo. Instituto de Investigaciones en Educación en las Ciencias Experimentales (FFHA-UNSJ). Disponible en: http://www.feeye.uncu.edu.ar/web/posjornadasinve/area3/Didactica\%20de\%20 la\%2oeducacion\%20superior/073\%20-\%20Lucero\%20-\%20Mendoza.pdf [Fecha de consulta: agosto 8 de 2012].

Makuc, M. 2008. Teorías implícitas de los profesores acerca de la comprensión de textos. Revista Signos 68: 403-422. Disponible en: http://www.scielo.cl/pdf/signos/v41n68/ art03.pdf [Fecha de consulta: agosto 9 de 2012].

Martínez, E., Díaz, N., Rodríguez, D. 2011. El andamiaje asistido en procesos de comprensión lectora en universitarios. Educación y Educadores 3: 531-555. Disponible en: $\quad$ http://educacionyeducadores.unisabana.edu.co/index.php/eye/article/ view/2044/2572 [Fecha de consulta: julio 3 de 2012].

Maturano, C., Soliveres, M. y Macías, A. 2002. Estrategias cognitivas y metacognitivas en la comprensión de un texto de ciencias. Enseñanza de las Ciencias 3: 415-425. Disponible en: https://docs.google.com/viewer?a=v\&q=cache:WmbxaYyA7KYJ:www. raco.cat/index.php/Ensenanza/article/download/21831/21665+27.+Maturano,+Carla+ In\%C3\%A9s, +Soliveres, +Mar\%C3\%ADa+Amalia+Y+Mac\%C3\%ADas,+Ascensi\%C3\%B3n+(2 002).+Estrategias+cognitivas+y+metacognitivas+en+la+comprensi\% $C 3 \% B 3 n+d e+u n+$ texto+de+ciencias.+Instituto+de+investigaciones+en+educaci\%C3\%B3n+en+las+cienc ias+experimentales.+Facultad+de+filosof\%C3\%ADa,+humanidades+y+artes.+Universi dad+nacional+de+san+juan.+Av.+J.i.+De+la+roza,+230+oeste.+5400+san+juan.\&hl=e n\&gl=co\&pid=bl\&srcid=ADGEESjpUTO45vDUcwTXGtE6v_IXizCnjmWoP1CiViogQf_Ge 7tDo9SoKRiQmNUz3O3M9YsUooWDIAsnyPAyonYpknMbJCSUzkP8xQQZaGH62liAgtYPGyFuoD45V_runihJHNyyBh1\&sig=AHIEtbTb2f78gIIYYf2edoXjCdmwj7VySw [Fecha de consulta: agosto 6 de 2012]

Miljanovich, M., Huerta, R., Atalaya, M., Evangelista, D., Chávez, M., Castañeda, F., Paredes, M., Woll, P. 2007. Módulo recuperativo de comprensión lectora para estudiantes universitarios. Revista IIPSI 2: 105-110. Disponible en: http://sisbib.unmsm. edu.pe/BVRevistas/Investigacion_Psicologia/v10_n2/pdf/a07v10n2.pdf [Fecha de consulta: agosto 17 de 2012].

Moyano, E. 2010. Escritura académica a lo largo de la carrera: Un programa Institucional. Signos 74: 465-488. Disponible en: http://www.scielo.cl/scielo.php?pid=S071809342010000500004\&script=sci_arttext [Fecha de consulta: junio 29 de 2012]. 
Narváez, E. \& Cadena, S. 2009. La enseñanza de la lectura académica: un objeto de formación docente. Lectura y Vida 30:56-67. Disponible en: http://www.lecturayvida. fahce.unlp.edu.ar/numeros/a30n1/30_01_Narvaez.pdf/view [Fecha de consulta: julio 6 de 2012].

Narváez, E. y Chois, M. 2012. La lectura y la escritura académica en la universidad colombiana: desafios para la orientación de politicas educativas en el marco de una investigación interuniversitaria. Lenguaje 40 (2): 285-313. Disponible en: http:// revistalenguaje.univalle.edu.co/index.p hp?seccion=REVISTA\&revista=40-2 [Fecha de consulta: julio 14 de 2012].

Novo, M., Tenca, L. Escalarea, L. 2008. Imágenes autorales en el aprendizaje de la escritura adadémica. Letra Inversa 4: 61-73. Disponible en: http://www.ifdcvm.edu.ar/ letrainversa/letra_inversa_04.pdf\#page=61 [Fecha de consulta: agosto 21 de 2012].

Ochoa, S. \& Aragón, L. 2005. Comprensión lectora y funcionamiento meta-cognitivo en estudiantes universitarios. Universitas Psychologica 4 (2): 179-196. Disponible en: $\quad$ http://www.scielo.unal.edu.co/scielo.php?script=sci_arttext\&pid=S165792672005000200006\&lng=es\&nrm= [Fecha de consulta: agosto 21 de 2012].

Oliva, A. 1999. Aprender a leer, para aprender leyendo, una experiencia en el nivel superior. La Lectura 3. Disponible en: http://aal.idoneos.com/index.php/Revista/ A\%C3\%B10_3_Nro._2/Comprensi\%C3\%B3n_de_textos_cient\%C3\%ADficos [Fecha de consulta: julio 19 de 2012].

Ortega, M. \& Andrade, N. 2007. La participación docente en la comprensión de la lectura en estudiantes universitarios. Revista electrónica de Psicología 4: 80-97. Disponible en: http://dgsa.uaeh.edu.mx/revista/psicologia/IMG/pdf/No.4-4.pdf [Fecha de consulta: junio 20 de 2012].

Paradiso, J.1996. Comprensión de textos expositivos: estrategias para el aula. Anales de Psicología 12(2): 167-177. Disponible en: http://www.um.es/analesps/v12/v12_2/05-12-2. pdf [Fecha de consulta: junio 23 de 2012].

Parra, C. 2012. Lectura, escritura y cultura académica en la universidad. Avances de investigación. Redlecturas. 4. Disponible en: http://www.faceducacion.org/ redlecturas4/?q=node/45 [Fecha de consulta: junio 22 de 2012].

Patiño, L. 2010. La escritura académica en la formación del docente universitario. Revista Educación y Pedagogía 18(46): 125-133. Disponible en: http://aprendeenlinea. udea.edu.co/revistas/index.php/revistaeyp/article/viewFile/6940/6353 [Fecha de consulta agosto 24 de 2012].

Perilla, A., Rincón, G., Gil, J., Salas R. 2004. El mejoramiento de los procesos de comprensión de textos académicos en el ámbito universitario. Revista Lenguaje 32: 160182. Disponible en: http://bibliotecadigital.univalle.edu.co/bitstream/10893/2741/2/ Lenguaje\%2032\%2cp.159-182\%2c2004.pdf [Fecha de consulta: julio 24 de 2012]. 
Piacente, T. \& Tittarelli, A. 2006. Comprensión y producción de textos en alumnos universitarios: la reformulación textual. Orientación y Sociedad 6: 1-6. Disponible en: http://www.scielo.org.ar/pdf/orisoc/v6/v6a05.pdf [Fecha de consulta: julio 3 de 2012].

Rapetti, M. \& Vélez, G. 2012. Leer para aprender y aprender a leer en la universidad: entre las lecturas estéticas y eferentes. Revista Iberoamericana de Educación Superior 7: 113-128. Disponible en: http://ries.universia.net/index.php/ries/article/view/125/pdf_25 [Fecha de consulta: junio 25 de 2012].

Reyes, S., Fernández-Cárdenas, J. \& Martínez Martínez, R. 2013. Comunidades de blogs para la escritura académica en la enseñanza superior: un caso de innovación educativa en México. Revista mexicana de investigación educativa, 18(57): 507-535. Disponible en: http://www.scielo.org.mx/scielo.php?script=sci_arttext\&pid=S140566662013000200009\&lng=es\&tlng=es. [Fecha de consulta: 31 de enero de 2014].

Rinaudo, M. 1991. Estudiar y aprender. Investigaciones sobre la comprensión y aprendizaje de textos. La Educación: Revista Interamerica de Desarrollo Educativo 126128 (1.13). Disponible en: http://fisica.usach.cl/ jlay/html/pdf/estudiaryaprender.pdf [Fecha de consulta: agosto 3 de 2012].

Rincón, G. \& Gil, J. 2010. Las prácticas de lectura y de escritura académicas en la Universidad del Valle: tendencias. Lenguaje 38 (2): 387-419. Disponible en: http:/l dintev.univalle.edu.co/revistasunivalle/index.php/Lenguaje/article/view/379/387 [Fecha de consulta: 18 de julio de 2012].

Rincón, G. y Gil, J. 2010. Estado de tendencias En las prácticas de lectura y escritura académicas en la Universidad del valle. Universidad del Valle. Disponible en: http:// sintesis.univalle.edu.co/2010/septiembre/lectura.pdf [Fecha de consulta: agosto 5 de 2012].

Rincón, G., Narváez, E. y Roldan, A. 2004. Universidad del Valle 8. Enseñar a comprender textos en la universidad: ¿Qué y cómo se está haciendo? Lenguaje 32:183211. Disponible en: http://bibliotecadigital.univalle.edu.co/bitstream/10893/2742/3/ Lenguaje\%2032,p.183-211,2004.pdf [Fecha de consulta: agosto 5 de 2012].

Rivera, M. 2003. Estrategias de lectura para la comprensión de textos escritos: el pensamiento reflexivo y no lineal en alumnos de educación superior. Umbral 12: 1-14 .Disponible en: http://www.farq.edu.uy/estructura/catedras/construccion/ construccion1/del\%2opensamiento\%2olineal\%20al\%20pensamiento\%2oreflexivo.pdf [Fecha de consulta: agosto 9 de 2012].

Rodríguez, J. 2007. Leer y escribir en primer año de la universidad: La mirada crítica de los docentes frente a las dificultades de los Ingresantes. Primeras Jornadas de Lectura y Escritura. Lectura y escritura criticas: perspectivas múltiples (1-8). Disponible en: http://www.filo.unt.edu.ar/jorn_unesco/cd/PO\%2071\%20RODRIGUEZ\%20Judith.pdf [Fecha de consulta: junio 5 de 2012]. 
Rolando, L., Bidiña, A., Zerillo, A., Pidoto, A., Espelta, M., Callegaro, A. \& Motta, M. 2002. La lectura en la universidad: de la comprensión a la interpretación. Universidad Nacional de La Matanza Programa de Incentivos. Disponible en: http://www. filo.unt. edu.ar/jorn_unesco/cd/PO\%2018\%20BIDINA.pdf [Fecha de consulta: julio 21 de 2012].

Sabaj, M. \& Ferrari, O. 2005. La comprensión de textos especializados en sujetos con formación profesional diferenciada. Pontificia Universidad Católica de Valparaíso Cátedra Unesco para la lectura y la escritura. Disponible en: http://www2.udec.cl/ catedraunesco/ogSABAJ\&FERRARI.pdf [Fecha de consulta: agosto 5 de 2012].

Sánchez, E., Orrantia, J. \& Rosales, J. 1992. Cómo mejorar la comprensión de textos en el aula. Comunicación, Lenguaje y Educación 14: 89-112. Disponible en: http://dialnet. unirioja.es/servlet/articulo?codigo=126250 [Fecha de consulta: agosto 29 de 2012].

Serrano, S. 2008. El desarrollo de la comprensión crítica en los estudiantes universitarios: hacia una propuesta didáctica. Educere: Artículos Arbitrados 42: 505-514. Disponible en: http://www.saber.ula.ve/bitstream/123456789/26307/1/articulo10.pdf [Fecha de consulta: agosto 25 de 2012].

Silvera, A., Collante, C., Arazona T., Ortiz, M. 2003. La competencia lectora de los estudiantes universitarios: un estudio para diseñar Estrategias dirigidas a su perfeccionamiento. Revista Psicogente. 11: 35-42.Disponible en: http://portal. unisimonbolivar.edu.co:82/rdigital/psicogente/index.php/psicogente/article/ viewFile/64/76 [Fecha de consulta: junio 30 de 2012].

Sosa, S. y Loss, T. 2007. La comunicación académica escrita: representaciones de Estudiantes universitarios sobre el sujeto destinatario. Facultad de Lenguas. Universidad Nacional de Córdoba. Disponible en: http://www.filo.unt.edu.ar/jorn_unesco/cd/ PO\%2077\%20SOSA\%20Y\%20LOSS.pdf [Fecha de consulta: agosto 5 de 2012].

Tolosa, A. \& Zerpa, C. 2009. Efecto de un programa de comprensión de textos de contenido moral en las estructuras de razonamiento moral de estudiantes del primer año del Ciclo Diversificado. Revista de Pedagogía 30(87): 247-280. Disponible en: http://biblioteca. universia.net/html_bura/ficha/params/title/efecto-programa-comprension-textoscontenido-moral-estructuras-razonamiento-moral-estudiantes/id/54639658.html [Fecha de consulta: junio 25 de 2012].

Uribe, Á. \& Martínez, Z. 2008. Prácticas de lectura y escritura académicas en la Universidad Colombiana. Magis 3 (6): 317-341. Disponible en: http://revistas.javeriana. edu.co/index.php/MAGIS/article/view/3543/2647 [Fecha de consulta: julio 3 de 2012]. 